\section{Acute bronchial obstruction following esophageal stent implantation for variceal bleeding}
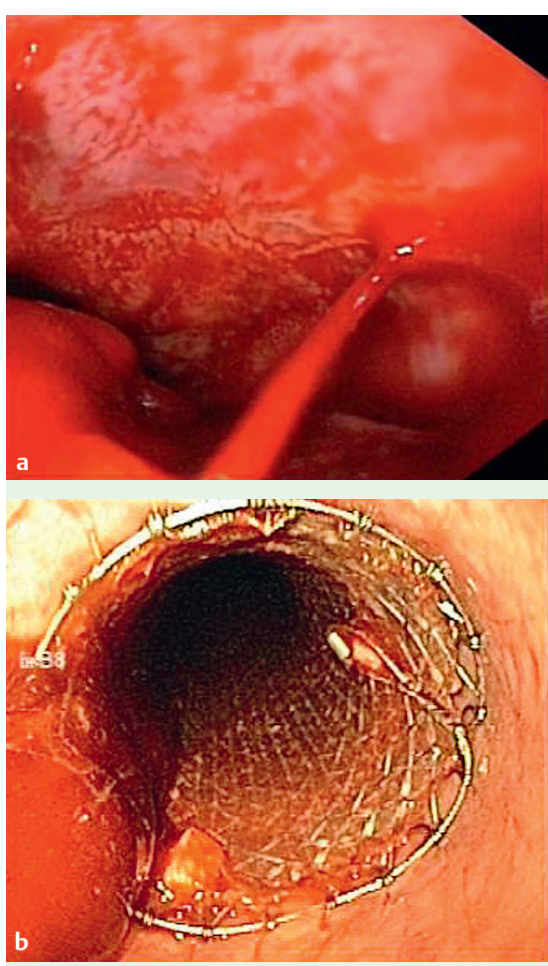

Fig. 1 a Bleeding esophageal varix located directly above the esophagogastric junction. b Self-expanding metal stent in the esophagus immediately after implantation.

A 59-year-old man with hilar cholangiocarcinoma and subsequent thrombosis of the intrahepatic portal vein presented with acute upper gastrointestinal hemorrhage. Esophagogastroduodenoscopy revealed acute bleeding from distal esophageal grade III varices ( $\bullet$ Fig. 1 a).

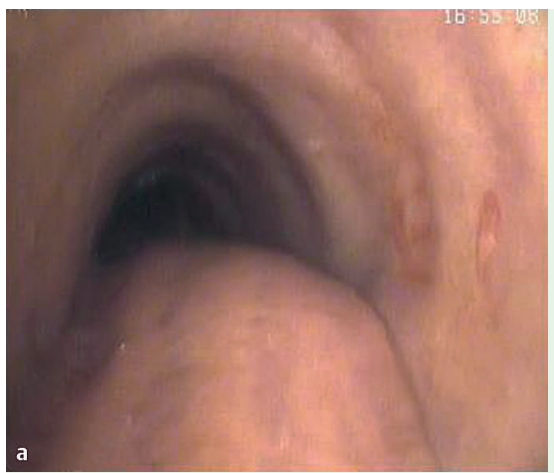

Variceal band ligation was unsuccessful, and the patient rapidly developed respiratory failure because of aspiration of regurgitated blood. After intubation and artificial ventilation, placement of a nitinol self-expanding metal stent (SX-Ella Danis, Hradec Králové, Czech Republic) immediately stopped the hemorrhage, as also confirmed endoscopically 1 day later ( 1 Fig. 1 b)

The post-procedure clinical course was uneventful; artificial ventilation was continued because of aspiration pneumonia. However, after 6 days, respiratory parameters deteriorated rapidly due to severe bronchial obstruction. Endotracheal bronchoscopy revealed profound narrowing of the proximal left main bronchus near the main carina because of extraluminal compression ( $\bullet$ Fig. 2 a).

Stent-related compression of the left main bronchial wall was verified by computed tomography (CT) (॰ Fig. 2 b). Therefore, endoscopic stent removal was considered to be the only therapeutic option and was performed, resulting in resolution of the bronchial obstruction. Although no further variceal bleeding occurred after stent removal, 7 days later the patient died because of liver failure.

We describe an important complication of therapeutic self-expanding metal stent implantation for esophageal variceal bleeding. This stent consists of a plasticcovered nitinol mesh (length $135 \mathrm{~mm}$; diameter $25 \mathrm{~mm}$; proximal and distal flare $30 \mathrm{~mm}$ ). Its high radial expansion force enables compression of (bleeding) esoph- ageal varices. This stabilizes the situation for $\leq 7$ days (maximum duration of use as recommended by the manufacturer) before applying further therapeutic measures [1]. Airway malignancy is a contraindication, but bronchial compression in an otherwise unaffected respiratory system has not been reported. A few authors have reported rapid respiratory dysfunction after stent placement [2-4], but there are no reports on specific complications related to use of the stent described here.

We recommend careful selection of patients with respiratory impairment, and, in case of deterioration after stent implantation, to consider and rapidly treat airway compression, e.g. by immediate stent removal.

Endoscopy_UCTN_Code_TTT_1AO_2AD Endoscopy_UCTN_Code_TTT_1AO_2AZ

A. Dechene', M. Adamzik², G. Gerken ${ }^{1}$, A. Canbay ${ }^{1}$

1 Department of Gastroenterology and Hepatology, University Hospital Essen, Essen, Germany

2 Department of Anesthesia, University Hospital Essen, Essen, Germany

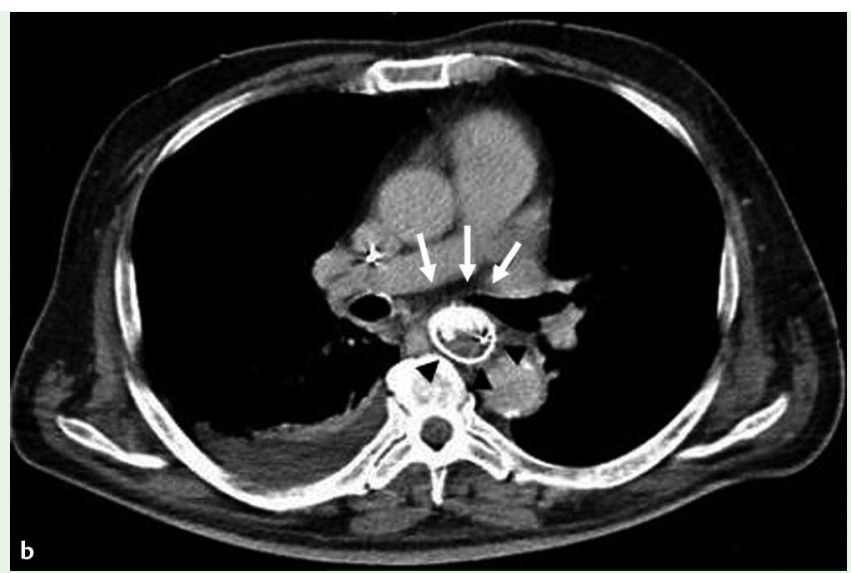

Fig. 2 Bronchoscopic view of left main bronchus with luminal impression of the esophageal stent. $\mathbf{b}$ CT scan of the thorax at the level of the tracheal bifurcation showing compression of the left main bronchus (white arrows) by the esophageal stent (black arrowheads). 


\section{References}

1 Hubmann R, Bodlaj G, Czompo M et al. The use of self-expanding metal stents to treat acute esophageal variceal bleeding. Endoscopy 2006; 38: $896-901$

2 Farivar AS, Vallieres E, Kowdley KV et al. Airway obstruction complicating esophageal stent placement in two post-pneumonec- tomy patients. Ann Thorac Surg 2004; 78: e22-e23

3 Dasgupta A, Jain P, Sandur S et al. Airway complications of esophageal self-expandable metallic stent. Gastrointest Endosc 1998; 47: $532-535$

4 Libby ED, Fawaz R, Leano AM et al. Airway complication of expandable stents. Gastrointest Endosc 1999; 49: 136-137
Bibliography

DOI $10.1055 / \mathrm{s}-0028-1119725$

Endoscopy 2009; 41: E146 - E147

(c) Georg Thieme Verlag KG Stuttgart · New York . ISSN 0013-726X

\section{Corresponding author}

\section{A. Canbay, MD}

University Hospital Essen

Hufelandstr. 55

45122 Essen

Germany

Fax: +49-201-7235719

ali.canbay@uni-due.de 